\title{
Diabetes and exocrine pancreatic insufficiency in E2F1/E2F2 double-mutant mice
}

\author{
Ainhoa Iglesias, ${ }^{1}$ Matilde Murga, ${ }^{1}$ Usua Laresgoiti, ${ }^{1}$ Anouchka Skoudy, ${ }^{2}$ \\ Irantzu Bernales, ${ }^{1}$ Asier Fullaondo, ${ }^{1}$ Bernardino Moreno, ${ }^{3}$ José Lloreta, ${ }^{4}$ \\ Seth J. Field, ${ }^{5}$ Francisco X. Real, ${ }^{2}$ and Ana M. Zubiaga ${ }^{1}$
}

\begin{abstract}
${ }^{1}$ Department of Genetics, Physical Anthropology and Animal Physiology, Faculty of Sciences, University of the Basque Country, Bilbao, Spain. ${ }^{2} U n i t a t ~ d e$ Biologia Cellular I Molecular, Institut Municipal d'Investigació Mèdica, Universitat Pompeu Fabra, Barcelona, Spain. ${ }^{3 B}$ Basque Institute for Agrarian Research and Development (NEIKER), Derio, Spain. ${ }^{4}$ Servei de Patologia, Hospital del Mar, Barcelona, Spain. ${ }^{5}$ Division of Signal Transduction, Beth-Israel Deaconess Hospital and Division of Endocrinology, Massachusetts General Hospital, Harvard Medical School, Boston, Massachusetts, USA.
\end{abstract}

\begin{abstract}
E2F transcription factors are thought to be key regulators of cell growth control. Here we use mutant mouse strains to investigate the function of E2F1 and E2F2 in vivo. E2F1/E2F2 compound-mutant mice develop nonautoimmune insulin-deficient diabetes and exocrine pancreatic dysfunction characterized by endocrine and exocrine cell dysplasia, a reduction in the number and size of acini and islets, and their replacement by ductal structures and adipose tissue. Mutant pancreatic cells exhibit increased rates of DNA replication but also of apoptosis, resulting in severe pancreatic atrophy. The expression of genes involved in DNA replication and cell cycle control was upregulated in the E2F1/E2F2 compound-mutant pancreas, suggesting that their expression is repressed by E2F1/E2F2 activities and that the inappropriate cell cycle found in the mutant pancreas is likely the result of the deregulated expression of these genes. Interestingly, the expression of ductal cell and adipocyte differentiation marker genes was also upregulated, whereas expression of pancreatic cell marker genes were downregulated. These results suggest that E2F1/E2F2 activity negatively controls growth of mature pancreatic cells and is necessary for the maintenance of differentiated pancreatic phenotypes in the adult.
\end{abstract}

\section{Introduction}

Normal cellular proliferation and differentiation require the transcriptional control of a large number of genes. Aberrant expression of a single transcriptional regulator may result in neoplasia or in altered tissue differentiation and development (1). E2F transcription factors, targets of retinoblastoma family members, regulate the expression of many genes implicated in cell growth and differentiation (2-4). E2F was first found to be important for the transcriptional activation of genes involved in G1/S progression and DNA replication (2-4). Recently, DNA microarray analyses have extended the list of potential E2F target genes to include DNA damage checkpoint and repair genes, factors involved in chromatin assembly/condensation, chromosome segregation, mitotic spindle checkpoint, apoptosis, differentiation, and development (5-8).

The E2F family is composed of six members (E2F1-6) that heterodimerize with DP proteins to form a DNA-binding transcriptional activator (2). Although much has been learned about the biological properties of the E2F transcription factors, the precise roles of each individual member, as well as the level of redundancy among them, need to be resolved. Chromatin immunoprecipitation assays in cultured cells have not detected the selective association of individual E2F-DP complexes to a variety of known E2F-regulated promoters $(9,10)$, suggesting that there is little target specificity and much redundancy among the E2F family

Nonstandard abbreviations used: E2F1/E2F2 double-deficient (DKO); expressed sequence tag (EST); microkatal ( $\mu$ kat); murine embryonic fibroblast (MEF).

Conflict of interest: The authors have declared that no conflict of interest exists.

Citation for this article: J. Clin. Invest. 113:1398-1407 (2004).

doi:10.1172/JCI200418879. members. Conversely, analysis of mouse strains carrying targeted mutations for individual $E 2 F$ genes have shown that loss of individual E2Fs can have distinct developmental and physiological consequences, implying the existence of specific target genes for each E2F. $E 2 F 1^{-/-}$mice display defective thymocyte apoptosis and impaired thymic negative selection, increased tumor susceptibility, exocrine gland dysplasia, and testicular atrophy (11-14). E2F2 $2^{-/}$mice exhibit increased proliferation of hematopoietic cells and frequently develop autoimmunity and tumors $(15,16)$. $E 2 \mathrm{F3}^{-/-}$mice exhibit a partially penetrant embryonic lethality, and the surviving animals die prematurely with signs typical of congestive heart failure (17). E2F4 ${ }^{-/-}$mice are runts and show defective maturation of particular cell types $(18,19)$, and E2F5mice develop nonobstructive hydrocephalus as newborns (20). Importantly, a recent DNA microarray-based study comparing gene expression in $\mathrm{E}_{2} \mathrm{F1}^{-/-}$and WT mice identified E2F1-specific target genes whose promoters were bound exclusively by E2F1 (21), thus implying target gene specificity and unique functions among the E2Fs. Remarkably, these promoters do not contain consensus E2F sites, suggesting that E2F1 is recruited to the promoter regions either by binding to a novel sequence element or by interaction with another DNA-binding protein.

Studies of mice carrying two or more mutant E2F loci simultaneously have shown that the development of a variety of tissues is mainly dependent on the dosage of individual E2F members, but these studies have also provided support for the specificity model for certain E2F-dependent functions. In E2F1/E2F3-compound mutant mice, almost all of the developmental and age-related defects arising in the individual $E 2 \mathrm{~F}^{-/-}$or $E 2 \mathrm{~F}^{-/-}$mice were exacerbated by the mutation of the other E2F, suggesting that the 
development of a wide variety of tissues shows a critical dependence on the dosage of E2F1 and E2F3 (22). The one exception to this rule was the incidence of tumor formation, which did not increase in double-mutant mice compared with E2F1 single-mutant mice. The combined mutation of E2F3 with E2F1, E2F2, or both results in progressive reductions in cell cycle reentry of murine embryonic fibroblasts (MEFs), and triple-mutant MEFs are completely unable to proliferate (23). Reexpression of E2F3 completely restores proliferation, however, whereas E2F1 or E2F2 reexpression only partially rescues the growth defect of triple $\mathrm{KO}$ cells. The interpretation of these results has been that E2F1, E2F2, and E2F3 may contribute unique functions in cell-cycle control $(4,23)$. An extensive analysis of these mutant mouse models should shed light on the unique and redundant roles of each, individual E2F member in vivo.

Our previous studies with $E 2 \mathrm{F1}^{-/-}$and $E 2 \mathrm{~F}^{-/-}$mice led to the demonstration that these genes play unique biological roles in $\mathrm{T}$ lymphocyte homeostasis $(14,15)$. We now report that E2F1 and E2F2 are required for normal pancreatic function. E2F1/E2F2 doubledeficient (DKO) mice develop diabetes as well as exocrine gland dysfunction. Cell cycle analyses show a high turnover rate of E2F1/E2F2 double-mutant pancreatic cells compared with WT cells, characterized by an increased DNA replication rate and an increased apoptosis rate. The analysis of the E2F1/E2F2-responsive genes that are deregulated in double-mutant mice suggests a critical role for E2F1 and E2F2 gene activity in controlling proliferation and maturation of pancreatic cells. This identifies a novel function for these E2F family members in the maintenance of end-organ differentiation.

\section{Methods}

Metabolic studies. Mice were maintained on a normal light/dark cycle in cages with microisolator lids and were genotyped as previously described $(14,15)$. All procedures were approved by the University of the Basque Country Animal Care and Use Committee.

Blood glucose levels were determined from blood taken from mouse tails, using an automatic glucose monitor. Blood for insulin, glucagon, amylase, ions, and metabolite levels was taken by retroorbital bleeds. Insulin levels were measured in serum by ELISA (ALPCO Diagnostics, Windham, New Hampshire, USA), glucagon was measured in plasma by radioimmunoassay (ALPCO Diagnostics), and amylase activity was measured in serum (BioSystems S.A., Barcelona, Spain), according to the manufacturer's instructions. Amylase activity results were expressed as microkatals ( $\mu$ kats) of enzymatic activity per liter of serum. For insulin-tolerance tests, fasted (16 hour) diabetic animals received saline or human insulin intraperitoneally $(0.5 \mathrm{U} / \mathrm{kg}$ of body weight; Novo Nordisk A/S, Bagsvaerd, Denmark). Blood glucose levels were determined immediately before injection and at the indicated times. Results were expressed as percentages of initial blood glucose concentration.

Histology, ultrastructural analysis, and immunobistochemistry. Freshly collected tissues were fixed with $10 \%$ formalin in PBS and embedded in paraffin for staining with $\mathrm{H} \& \mathrm{E}$. For quantitation of acinar cell density, sections obtained from 7-day-old and 2-month-old male mice $(n=6 \mathrm{WT} ; n=4 \mathrm{E} 2 \mathrm{~F} 1$ null; $n=4 \mathrm{E} 2 \mathrm{~F} 2$ null; $n=6 \mathrm{E} 2 \mathrm{~F} 1 /$ E2F2 DKO) were stained with $\mathrm{H} \& \mathrm{E}$, and the total number of acinar cells per area $\left(200 \mu \mathrm{m}^{2}\right)$ was counted manually. In each animal, six randomly selected areas lacking any islets were used to calculate the average number of acinar cells per area.

For electron microscopy, small pancreas pieces were processed following standard procedures. A digitalized image-capture system was used to measure the size of secretory granules present in exo- crine and endocrine cells from WT mice, as well as granules present in cells containing mixed granule populations in DKO mice.

Immunohistochemical assays were performed on tissues fixed with $4 \%$ paraformaldehyde in PBS using an indirect immunoperoxidase-detection protocol (DAKO A/S, Glostrup, Denmark) after antigen retrieval, with autoclave treatment for 1 minute at $120^{\circ} \mathrm{C}$. $\mathrm{Ab}$ localization was determined using 3,3-diaminobenzidine in PBS. The Ab's used were rabbit mAb's to glucagon (1:5,000; ICN Biomedicals Inc., Irvine, California, USA), and $\alpha$-amylase ( $3 \mu \mathrm{g} /$ ml; Sigma-Aldrich, St. Louis, Missouri, USA), rabbit polyclonal Ab's to Pax-6 $(1: 4,000)$ and Pdx-1 (1:300), mouse mAb to insulin (1:3,000; Sigma-Aldrich), and mouse mAb to Isl-1 (1:4). Control immunostainings using the secondary $\mathrm{Ab}$ in the absence of the primary Ab were routinely performed.

In vivo S-phase labeling and apoptosis. For S-phase labeling, mice were injected intraperitoneally with $\mathrm{BrdU}(50 \mu \mathrm{g} / \mathrm{g}$ of body weight). Mouse organs were harvested 8 hours later and fixed in $10 \%$ formalin. Tissue sections were processed using the BrdU staining kit according to the manufacturer's instructions (Oncogene Research Products, Cambridge, Massachusetts, USA). Sections were counterstained with hematoxylin.

For in vivo apoptosis, pancreata and thymuses from WT, $E 2 \mathrm{F1}^{-/-}$, $\mathrm{E}_{2} \mathrm{~F}^{-/-}$, and E2F1/E2F2 double-mutant mice were harvested and processed for TUNEL, according to the instructions for the peroxidase Apoptag Kit (Intergen Co., Purchase, New York, USA).

Oligonucleotide microarrays. Total RNA was isolated from pancreatic tissue with the TRIzol reagent (Invitrogen Corp., Carlsbad, California, USA), according to the manufacturer's instructions, purified using the RNeasy kit (QIAGEN GmbH, Hilden, Germany), and electrophoresed on a denaturing agarose gel to examine for RNA integrity. Poly(A)+ RNA samples from 15-day-old pancreata ( $n=6$ for each genotype) were purified, and two pools were made from either WT or E2F1/E2F2 double-mutant mice. Each pool was a mix of RNA samples from three pancreata of the same genotype obtained from one female and two male mice. Target preparation, microarray hybridization, and transcriptome analysis were carried out with the Affymetrix GeneChip System (Affymetrix, Santa Clara, California, USA) at Medplant Genetics (Barakaldo, Spain). Briefly, each pool of purified mRNA samples was used to generate a cDNA with the SuperScript Choice System Kit (Invitrogen Corp.) according to the protocol of the Affymetrix Expression Analysis Technical Manual. The cDNA was then used to synthesize biotinylated cRNA with the BioArray High Yield RNA transcript-labeling kit (Enzo Diagnostics Inc., Farmingdale, New York, USA) according to the manufacturer's instructions, purified with the RNeasy Kit (QIAGEN GmbH), and fragmented. Target cRNA $(15 \mu \mathrm{g})$ was hybridized overnight to each oligonucleotide microarray (murine genome U74A V.2 Array; Affymetrix) containing probes for approximately 12,000 murine genes and expressed sequence tags (ESTs). Arrays were then washed and stained with streptavidin phycoerythrin. Fluorescent intensities were analyzed with GeneChip software (Affymetrix). We identified an upregulated or downregulated gene as one that was enhanced or reduced twofold or more in DKO samples relative to WT samples, respectively.

RT-PCR. Semi-quantitative RT-PCR was performed with total RNA isolated from pancreatic tissue, as described above. The cDNA was synthesized from $0.2 \mu \mathrm{g} / \mathrm{ml}$ of RNA using RT-PCR Superscript First-Strand Synthesis System for RT-PCR (Invitrogen Corp.), and all samples were diluted to the same final cDNA con- 

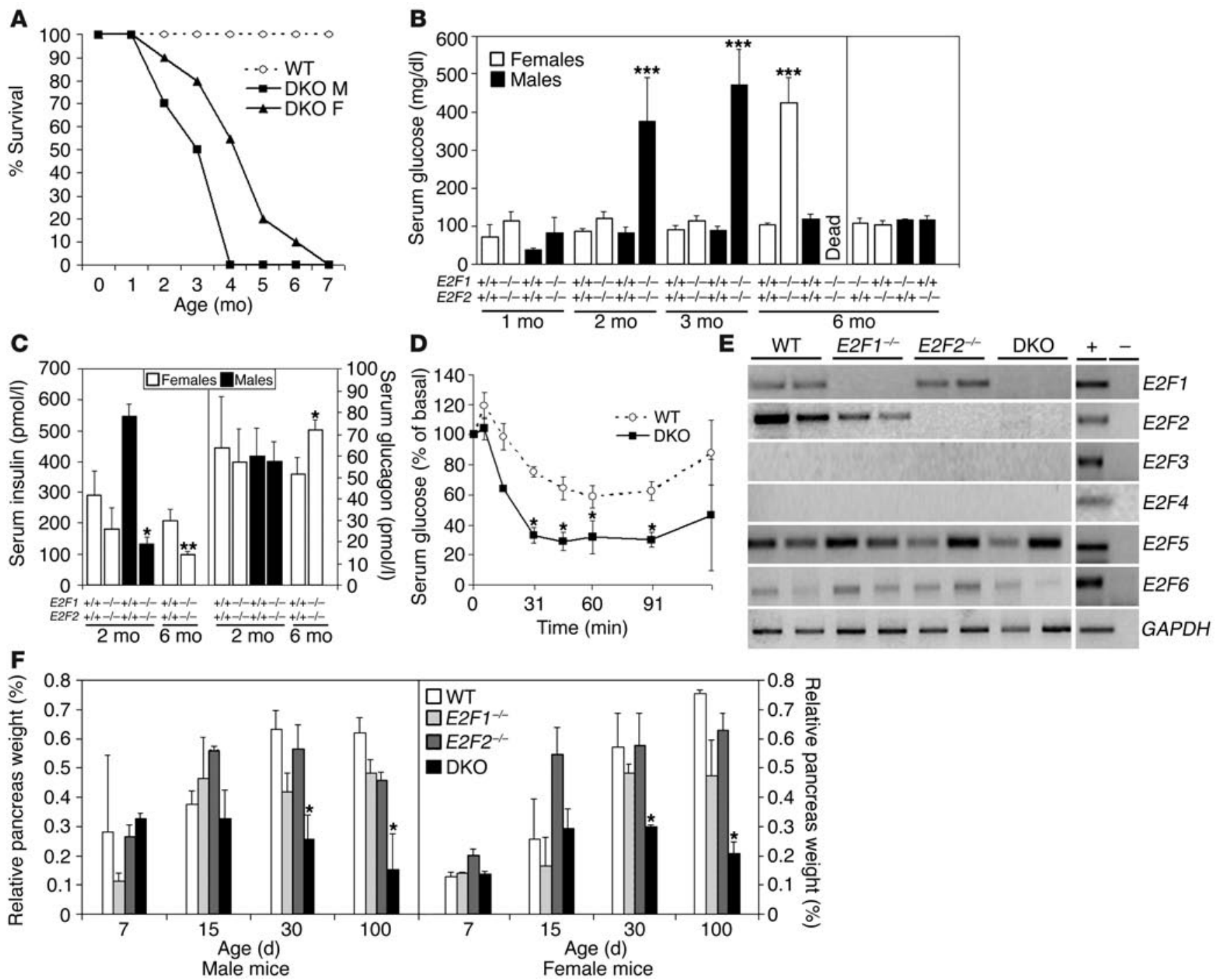

Figure 1

Hyperglycemia and pancreatic atrophy in E2F1/E2F2 compound-mutant mice. (A) Life span data obtained from WT ( $n=40)$, DKO male (DKO M; $n=25)$, and DKO female (DKO F; $n=25)$ mice were analyzed using a log-rank nonparametric test $(P<0.01)$ and expressed as Kaplan-Meier survival curves. (B) Spot blood glucose levels in WT, E2F1-- $E 2 F^{-1-}$, and DKO mice were determined at the indicated times. Results are the means \pm SEM for five to ten animals per sex and genotype. Data were analyzed using the two-tailed $t$ test $\left({ }^{* *} P<0.001\right)$. (C) Insulin and glucagon levels in serum of 2-month-old and 6-month-old WT and DKO mice $\left(n \geq 7\right.$ per genotype; ${ }^{*}<0.05$; $\left.{ }^{* \star} P<0.01\right)$. (D) Insulin-tolerance test was performed in starved animals following intraperitoneal injection of insulin into 5- to 6-month-old hyperglycemic female DKO mice $(n=8)$ or normoglycemic female WT controls $(n=7)$. Results are expressed as percentage of initial blood glucose concentration $\left({ }^{*} P<0.05\right)$. Similar results were obtained for males (not shown). (E) RT-PCR analysis of RNA samples from 2-week-old WT, E2F1--- $E 2 F 2^{-1-}$, and DKO ( $n=2$ per genotype) mice showing expression of E2F1, E2F2, E2F5, and E2F6 in the pancreas of WT mice (E2F3 and E2F4 expression was absent in pancreas). As shown, E2F5 and E2F6 were similarly expressed in the WT and DKO pancreas. The last row shows expression of an internal standard (GAPDH) amplified in a reaction parallel to the test genes. (F) Pancreas weight expressed as fraction of total body weight. Shown are means \pm SEM for four to six animals per genotype and sex at each time point analyzed. The nonparametric Mann-Whitney $U$ test was used for comparative analysis $\left({ }^{*} P<0.05\right)$.

centration. PCR was performed for 35-40 cycles, depending on the primer set, and we ensured linear amplification in all cases. PCR reactions were performed a minimum of two times. Primers for each target gene were selected within their respective coding regions, such that genomic DNA and unspliced pre-mRNA would amplify as larger fragments because of the presence of introns. Primer sequences are available upon request. Internal standards for every PCR (GAPDH) were included, and all data were normalized to the internal standard. PCR products were analyzed on $2 \%$ agarose gels and stained with ethidium bromide. As positive controls for E2F1, E2F2, and E2F3 expression, RNA obtained from activated lymphocytes was used. As positive controls for E2F4, E2F5, and E2F6 expression, RNA obtained from mouse embryonic fibroblasts was used. For each RT-PCR reaction, a negative control containing an RNA sample, but lacking the corresponding cDNA, was also included. Data were quantitated with a Molecular Imager (BioRad Laboratories Inc., Hercules, California, USA) using the Quantity One program. 

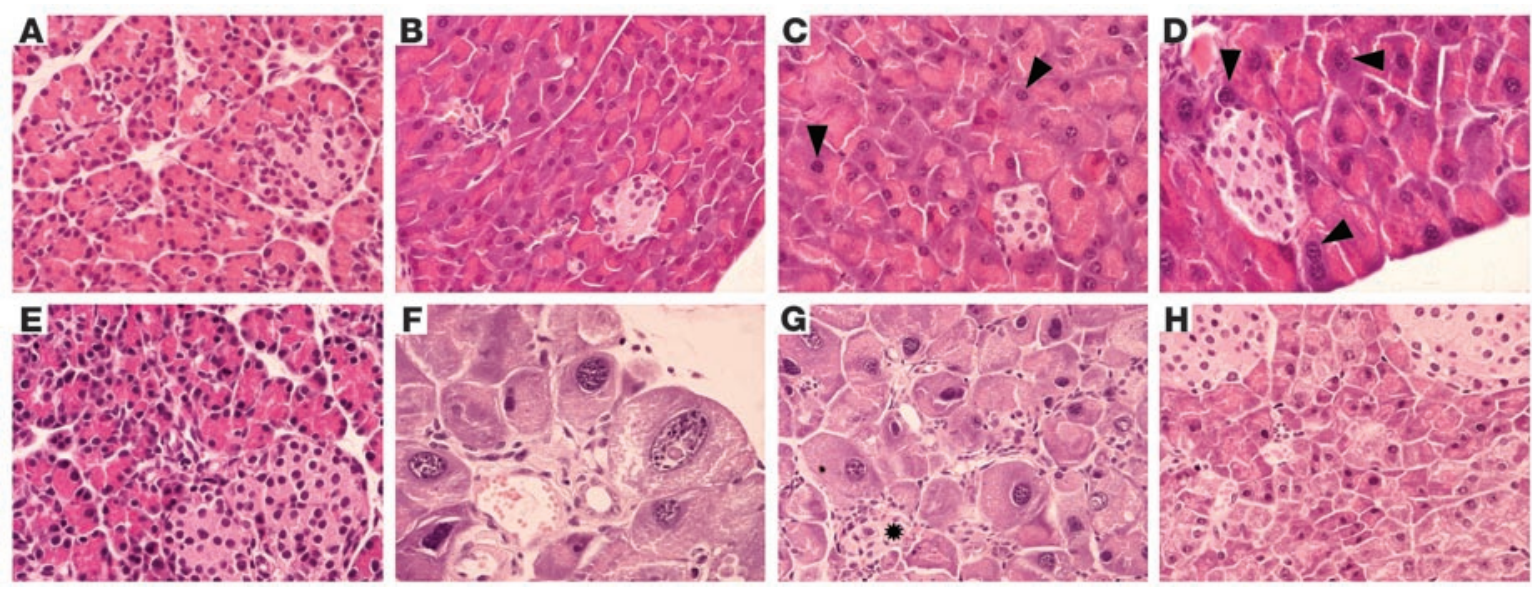

\section{Figure 2}

Pancreatic histopathology of E2F1/E2F2 double homozygotes. Pancreas sections obtained from E2F-deficient and WT animals stained with H\&E. (A and B) Representative pancreas section of 7-day-old (A) and 2-month-old (B) WT male mice. Magnification, $\times 600$. (C and D) Representative pancreas section of 2-month-old (C) and 6-month-old (D) E2F1-deficient male mice showing aberrantly large nuclei (marked with arrowheads). Magnification, $\times 600$. (E-G) Representative pancreas section of 7-day-old (E) and 2-month-old (F and $\mathbf{G})$ DKO male mice. (E and $\mathbf{F})$ Magnification, $\times 600$. (G) Magnification, $\times 400$. The acini and islets (marked with an asterisk) in the DKO pancreas are abnormal and have lost their typical tubular organization, which has been replaced by ductal structures. Note the dysplasic changes in acinar cells, characterized by hypertrophy and karyomegaly. (H) Representative pancreas section of a 2-month-old E2F2-deficient male mouse. Magnification, $\times 600$. The morphology of the pancreas in female E2F-deficient mice was essentially the same as the morphology of the pancreas in male counterparts (data not shown). The sections shown are representative examples from histology for six WT ( $n=3$ male, $n=3$ female), 15 DKO $(n=8$ male, $n=7$ female), six E2F1-/( $n=3$ male, $n=3$ female), and six E2F2-/- $(n=3$ male, $n=3$ female) mice.

\section{Results}

Development of diabetes in E2F1/E2F2 compound-mutant mice. The E2F1-deficient and E2F2-deficient mice have revealed unique roles for each of these genes. There may be functions that are common to multiple E2F family members, however, that are not revealed by single-gene mutations. To begin to understand the functions that are shared by E2F family members, we have generated mice deficient in both E2F1 and E2F2, two E2F family members that preferentially interact with the retinoblastoma protein. Crosses between $E 2 \mathrm{~F}^{+/-}$, $\mathrm{E} 2 \mathrm{~F}^{+/-}, \mathrm{E} 2 \mathrm{~F}^{-/-} / \mathrm{E} 2 \mathrm{~F}^{+/-}$, or $\mathrm{E} 2 \mathrm{~F} 1^{+/-} / \mathrm{E} 2 \mathrm{~F} 2^{-/-}$mice yielded viable $E 2 \mathrm{~F}^{-/-} / E 2 F 2^{-/-}$pups (DKO) at the expected Mendelian frequency. The E2F1/E2F2 DKO mice appeared overtly normal and similar in size to their littermates at birth, but their growth became increasingly retarded relative to their littermates throughout postnatal development. Although $E 2 \mathrm{Fi}^{-1-}$ / $E 2 \mathrm{~F}^{-/-}$mice were viable and outwardly appeared grossly normal, they had a severely limited life span (Figure 1A). Mean survival was $3.2 \pm 0.9$ months for males and $5.3 \pm 1.6$ months for females.

Adult DKO mice exhibited polyuria, polydipsia, and appeared lethargic prior to death. These features made us consider whether the mice were hyperglycemic. Indeed, random blood glucose levels increased to $471 \pm 94 \mathrm{mg} / \mathrm{dl}$ in 3-month-old DKO males near the mean time of death of these animals (normal level, $83 \pm 20 \mathrm{mg} / \mathrm{dl}$ ) (Figure 1B). Although females became hyperglycemic at an older age than males and had a longer life expectancy, the phenotype was fully penetrant in both sexes. Female E2F1/2 double-mutant mice remained normoglycemic for the first 2-3 months of age, but levels of glucose increased dramatically to over $400 \mathrm{mg} / \mathrm{dl}$ after the fourth month (Figure 1B). Consistent with these findings, urine glucose levels were also very high in DKO male and female mice by 1-2 and 4-5 months of age, respectively (data not shown). By contrast, random glucose levels in the serum of WT mice, single mutant $E 2 \mathrm{~F}^{-{ }^{--}}, \mathrm{E} 2 \mathrm{~F} 2^{-/-}$, or double heterozygote mice, were maintained at a level lower than $100 \mathrm{mg} / \mathrm{dl}$ throughout their lifetime (at least
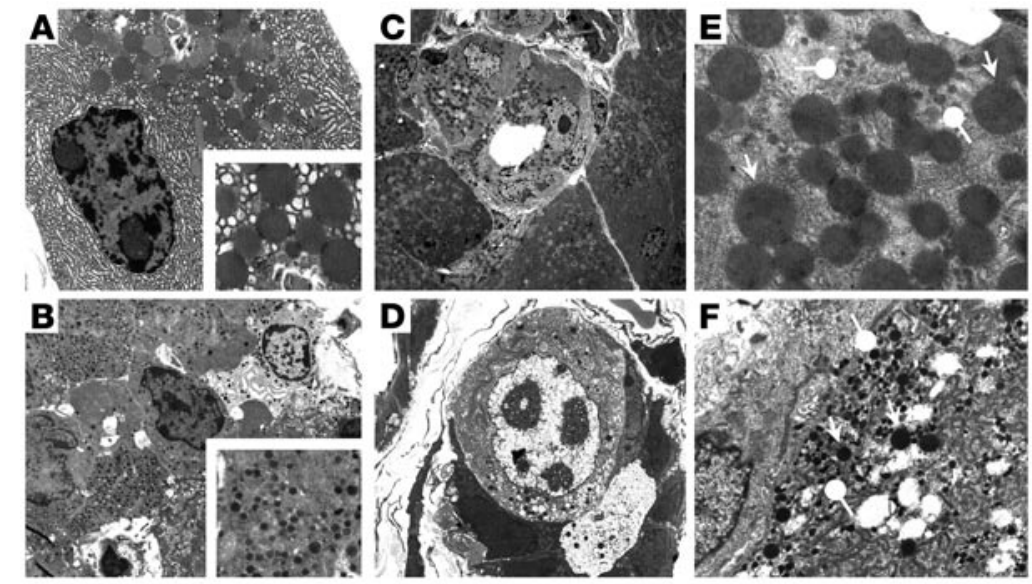

\section{Figure 3}

Ultrastructural analysis of E2F1/E2F2 double-homozygote pancreas sections. Exocrine (A) and endocrine (B) cells in pancreata from WT mice. Insets show the aspect of normal acinar and $\beta$ cell granules. Pancreas from a 3-month-old male DKO mouse (C-F). Ductal structures composed of transitional cells with ductal cell features, but containing zymogen granules (C), or both zymogen and endocrine granules, can be observed in DKO mice (D). Cells containing both types of granules (pointed arrows, acinar granules; round arrows, endocrine granules) ( $\mathbf{E}$ and $\mathbf{F})$. Original magnification: (A, B, E, F) ×3,400; (C) ×1,100; (D) $\times 2600$; insets in A and B, $\times 10,500$. 

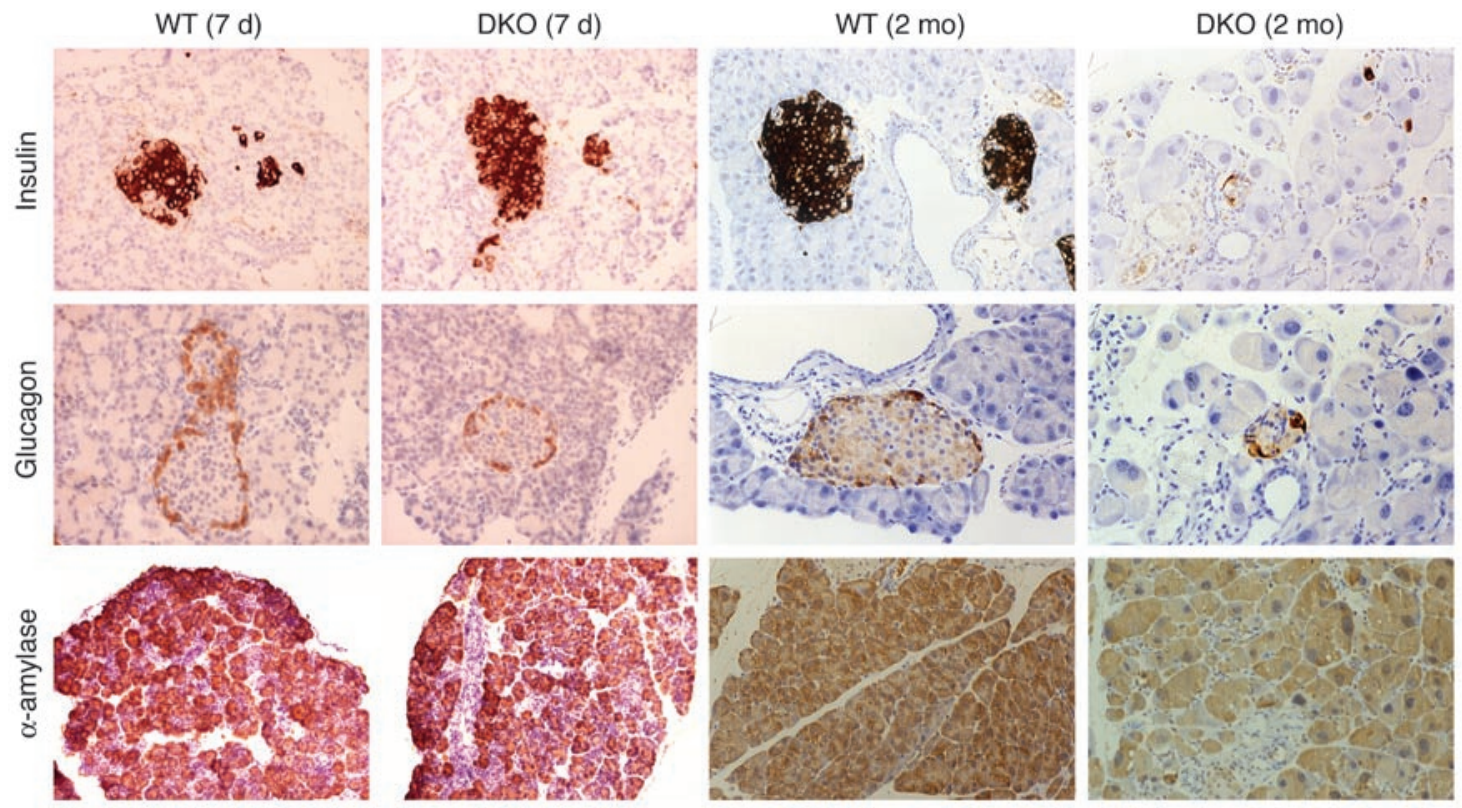

\section{Figure 4}

Immunohistochemical analysis of expression of pancreas-specific protein markers. Shown are representative pancreas sections of 7-day-old and 2-month-old WT and DKO male mice immunostained with Ab's to insulin, glucagon, and $\alpha$-amylase. A light hematoxylin counterstaining was performed in all sections (insulin and glucagon, $\times 400 ; \alpha$-amylase, $\times 200$ ). Similar results were obtained when DKO female mice were analyzed (data not shown).

beyond 1 year of age for each genotype) (Figure $1 \mathrm{~B}$ and data not shown). Concomitant with an increase in serum glucose levels, insulin levels in serum of hyperglycemic male and female doublemutant mice were reduced significantly (Figure 1C), suggesting that $E 2 F 1 / E 2 F 2$ double homozygotes develop insulin deficiency. Serum glucagon levels, on the other hand, remained similar in fasted hyperglycemic DKO mice relative to WT mice (Figure 1C). Furthermore, hyperglycemic DKO mice responded to injection of insulin with the expected reduction in serum glucose (Figure 1D). Taken together, these results suggest that the diabetic condition of E2F1/E2F2 double-deficient mice stems from loss of insulin production and is not due to insulin resistance.

Finally, urinalysis failed to detect an elevation in urinary ketones, and serum analysis failed to detect an elevated anion gap (data not shown), arguing that the cause of death in these mice is not diabetic ketoacidosis, as would be expected for selective pancreatic $\beta$ cell loss, but instead is the hyperosmolar hypernatremic nonketotic diabetic syndrome, more characteristic of complete loss of the pancreas.

Organ involution and cellular dysplasia in E2F1/2 double-deficient mice. To further define the phenotype of E2F1/2 DKO mice we first looked for anatomical and histological abnormalities. Loss of E2F1 and E2F2 in mice resulted in a marked reduction of size and cellularity of several organs, notably lymphoid organs, testicles, and salivary glands. For example, cell number in thymuses $\left(87.5 \times 10^{6} \pm 22.9\right.$ in DKO mice versus $169.6 \times 10^{6} \pm 54.5$ in WT mice, $P<0.05)$ and LNs $\left(10.7 \times 10^{6} \pm 4.2\right.$ in DKO mice versus $22.1 \times 10^{6} \pm 3.2$ in WT mice, $\left.P<0.05\right)$ was roughly reduced by half. Cell number in the spleen was reduced as much as twothirds $\left(24.3 \times 10^{6} \pm 13.0\right.$ in double mutants versus $77.3 \times 10^{6} \pm 18.1$ in WT, $P<0.05)$. The cellularity of all three lymphoid organs decreased further in older animals.
The most striking anatomical alteration was found in the pancreas: gross examination of this organ showed a remarkable involution over time in both male and female DKO mice (Figure 1F). The pancreas weight relative to body mass of 1- and 2-week-old DKO

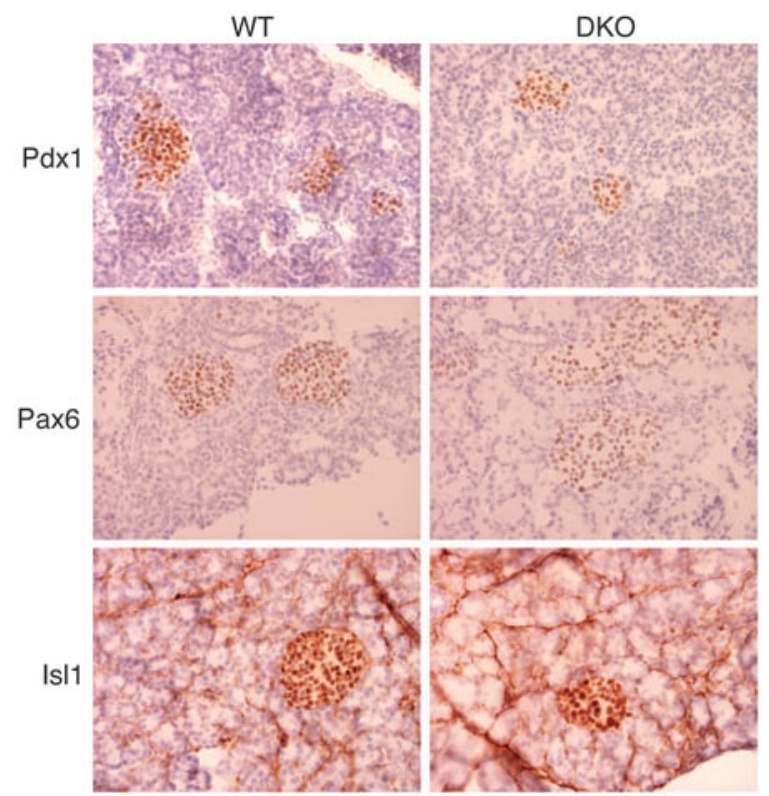

Figure 5

Immunohistochemical analysis of transcription factor expression in the pancreas. Shown are representative pancreatic islets of 7-day-old WT and DKO male mice immunostained with polyclonal Ab's to Pdx1, Pax6, and IsI1 $(\times 400)$. The expression of all three transcription factors in DKO mice was indistinguishable from age-matched WT mice. 


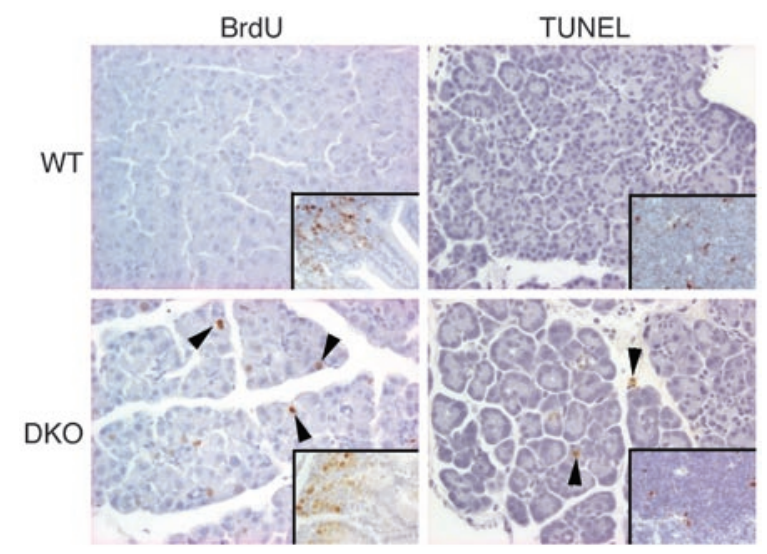

\section{Figure 6}

Increased proliferation and apoptosis in pancreas of E2F1/E2F2 compound-mutant mice. In vivo BrdU labeling (as described in Methods) identified pancreatic cells in S phase (appearing brown) in pancreata from 15-day-old mice. As shown, young DKO mice have an increased number of cells in S phase in the pancreas in vivo. Arrowheads in lower left panel indicate BrdU-positive nuclei. The presence of the expected labeling of intestinal crypt cells with BrdU in both genotypes (inset) serves as an internal control for the injection and incorporation of the tracer. Cell death was detected by TUNEL labeling in 15-day-old mice. Arrowheads in lower right panel denote apoptotic nuclei (appearing brown) detected by the TUNEL assay. Virtually no BrdU-positive or TUNEL-positive cells were detected in 15-day-old WT pancreata. The presence of the expected staining of thymocytes with TUNEL in both genotypes (inset) serves as an internal control for the experiment.

mice was similar to that of WT controls, but 1-month-old DKO animals exhibited a significant reduction in pancreas size compared with WT controls. This difference became even more pronounced as the animals aged. After 3 months of age, the pancreas of male and female DKO mice was almost completely atrophic, being replaced by fat, demonstrating that both endocrine and exocrine components were affected in these animals. Male DKO mice died shortly after the pancreas degenerated, whereas female DKO mice survived longer with an atrophic organ before death occurred. By contrast, no significant differences in pancreas size were noted in 6-month-old or younger $E 2 \mathrm{~F}^{-/-}$and E2F2 ${ }^{-/-}$single-mutant mice (Figure $1 \mathrm{~F}$ ), and only E2F1deficient mice over 6 months of age exhibited a substantial reduction in pancreas size relative to WT (ref. 12 and data not shown). Additionally, DKO mice had a distended colon containing soft, pale, fecal material, and the stools of DKO, but not those of WT, animals fed with a high-fat diet showed lipid droplets, suggesting fat malabsorption (data not shown). Furthermore, serum amylase levels in DKO animals younger than 10 weeks old were indistinguishable from those of WT, whereas they were reduced to half in mice older than 10 weeks of age $(23.7 \pm 4.9 \mu \mathrm{kat} / 1$ in WT mice compared with $11.4 \pm 0.7 \mu \mathrm{kat} / \mathrm{l}$ in DKO mice; $n=8$ per genotype; $P<0.02)$. Taken together, these results show that as DKO mice age, they develop severe endocrine and exocrine pancreatic insufficiency.

The overall histological appearance and cellular density of the pancreas of E2F1/E2F2 double-deficient mice was essentially normal until approximate- ly postnatal day 15 (Figure 2, A and E). Subsequently, the exocrine pancreas of male and female DKO mice lost its normal architecture and showed severe cellular abnormalities, including a lower number of acini and an increased number of ductal structures, fibrosis, and fat replacement. Furthermore, acinar cell hypertrophy with marked karyomegaly and nuclear inclusions were noted (Figure 2, $F$ and $G$ ). These changes were similar to, but more dramatic than, those observed in older $E 2 F 1^{-/-}$mice: a few large nuclei could be found in 2-month-old animals, but older mice showed a high proportion of hypertrophic cells with aberrantly enlarged nuclei (Figure 2, C and D). Consistent with these alterations, acinar cell density was lower in 2 -month-old $E 2 F 1^{-/-}$mice ( $55 \pm 13$ cells per area of $200 \mu \mathrm{m}^{2}$ in WT mice compared with $34 \pm 6$ in $E 2 \mathrm{~F}^{-/-}$mice). The reduction in acinar cell density was more dramatic in age-matched DKO mice (12 \pm 3 cells per area). By contrast, the pancreas of E2F2deficient mice appeared histologically normal in 2-month-old or older animals (Figure $2 \mathrm{H}$ ), and the average number of cells per area was similar to WT mice ( $53 \pm 4$ cells per area). The endocrine pancreas of DKO mice also became progressively atrophic. Islet number and size were overtly normal in 1- to 2-week-old DKO mice. A subsequent decrease in the size and number of islets was observed, however, and no islets could be found in older sick mice (Figure $2 \mathrm{G})$. The histologic alterations observed in the adult DKO pancreas were fully penetrant for both male and female mice.

Ultrastructural analysis of the pancreas from 2-month-old or older DKO mice showed hypertrophy, irregular shape, loss of polarity, lower zymogen granule content, dysplasia, and karyomegaly in acinar cells. Single acini often harbored transitional cells, characterized by ductal morphology but containing zymogen granules (Figure 3C). Cells containing zymogen-like and endocrine-like granules were also observed occasionally (Figure $3 \mathrm{D})$. In WT mice, the size of secretory granules in acinar cells was $727 \pm 184 \mathrm{~nm}(n=33$ granules measured, range 467-1,248) (Figure $3 \mathrm{~A})$, and the size of secretory granules in endocrine cells was $279 \pm 58$ ( $n=100$ granules measured, range 11-406) (Figure 3B). In WT mice there were no cells simultaneously containing both largesized and small-sized granules. By contrast, DKO mice exhibited cells containing two populations of granules: large, resembling
Table 1

DNA replication and apoptosis in pancreas of E2F-deficient mice

\begin{tabular}{|c|c|c|c|c|c|c|}
\hline & BrdU+ & TUNEL+ & & & & \\
\hline & 7 days & 15 days & 30 days & 7 days & 15 days & 30 days \\
\hline \multicolumn{7}{|l|}{ Males } \\
\hline $\begin{array}{l}\text { WT } \\
E 2 \mathrm{F1}^{-/-} \\
\text {E2F2-- } \\
\text { DKO }\end{array}$ & $\begin{array}{l}4.3 \pm 0.01 \\
4.7 \pm 0.01 \\
4.2 \pm 0.01 \\
4.5 \pm 0.01\end{array}$ & $\begin{array}{c}0.00 \\
1.2 \pm 1.0 \\
3.8 \pm 1.5^{\mathrm{C}} \\
14.4 \pm 3.6^{\mathrm{B}}\end{array}$ & $\begin{array}{c}0.00 \\
0.00 \\
1.2 \pm 0.8^{\mathrm{B}} \\
19.2 \pm 7.4^{\mathrm{A}}\end{array}$ & $\begin{array}{c}0.00 \\
1.6 \pm 0.4^{\mathrm{A}} \\
0.4 \pm 0.1^{\mathrm{B}} \\
0.4 \pm 0.1^{\mathrm{B}}\end{array}$ & $\begin{array}{c}0.1 \pm 0.0 \\
1.6 \pm 0.3^{\mathrm{A}} \\
0.00 \\
14.0 \pm 5.1^{\mathrm{A}}\end{array}$ & $\begin{array}{c}0.00 \\
0.00 \\
0.4 \pm 0.1 \\
3.8 \pm 2.2^{C}\end{array}$ \\
\hline \multicolumn{7}{|c|}{ Females } \\
\hline 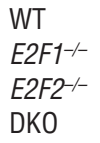 & $\begin{array}{l}4.2 \pm 0.02 \\
4.3 \pm 0.02 \\
4.5 \pm 0.01 \\
4.4 \pm 0.01\end{array}$ & $\begin{array}{r}0.6 \pm 0.3 \\
4.8 \pm 1.1^{\mathrm{B}} \\
11.6 \pm 3.1^{\mathrm{A}} \\
31.0 \pm 6.0^{\mathrm{A}}\end{array}$ & $\begin{array}{c}0.00 \\
1.8 \pm 0.7^{C} \\
5.3 \pm 1.2^{B} \\
11.2 \pm 3.6^{B}\end{array}$ & $\begin{array}{l}0.5 \pm 0.3 \\
1.1 \pm 0.5^{c} \\
0.5 \pm 0.1 \\
0.5 \pm 0.1\end{array}$ & $\begin{array}{l}0.1 \pm 0.1 \\
1.4 \pm 0.5^{\mathrm{C}} \\
0.5 \pm 0.3 \\
3.7 \pm 0.9^{\mathrm{A}}\end{array}$ & $\begin{array}{c}0.00 \\
1.3 \pm 0.5^{\mathrm{C}} \\
0.00 \\
2.4 \pm 1.6^{\mathrm{C}}\end{array}$ \\
\hline
\end{tabular}

Male and female mice of the indicated ages and genotypes were injected intraperitoneally with BrdU, and tissue sections were immunostained with a BrdUspecific $\mathrm{Ab}$. BrdU+ and TUNEL+ cell ratios were calculated as the mean \pm SEM per 1,000 cells. Six tissue sections per animal and three to five animals per genotype and sex were analyzed. ${ }^{A} P<0.001 ;{ }^{B} P<0.01 ;{ }^{C} P<0.05$. 
zymogen granules, ranging in size from 385 to $1,129(n=88)$, and small, endocrine-like granules, ranging in size from 97 to 256 $(n=107)$ (Figure 3, C-F). The fact there was no overlap in the size of both types of granules and that each type of granule had distinct electron density strongly suggests that they represent different entities. These results suggest that loss of E2F1 and E2F2 affects postnatal differentiation and maturation of the pancreatic cells, resulting in a cell type with ultrastructural features of both the endocrine and exocrine pancreas.

The distinct pancreatic phenotype that we found in E2F1/E2F2 double-deficient mice prompted us to examine the expression profiles of E2F family members in WT, E2F1-/, $E 2 F 2^{-/-}$, and E2F1/E2F2 double-mutant mice. As expected, E2F1 and E2F2 transcripts were detected in the pancreas of WT animals. Moreover, loss of E2F1 alone did not result in increased expression of E2F2 mRNA and vice versa (Figure 1E). E2F3 and E2F4 transcripts were undetectable in the pancreatic tissue of WT mice, and, importantly, E2F3 and E2F4 transcripts were also undetectable in E2F1 or E2F2 single-knockout or DKO mice. Finally, E2F5 and E2F6 expression levels in mutant animals were unaltered with respect to WT controls, arguing that the pancreatic phenotype of E2F1/E2F2 double-mutant mice does not result from altered expression of another E2F member.

Next, we analyzed expression of pancreatic differentiation markers by immunohistochemistry. Expression of insulin, glucagon, and $\alpha$-amylase was normal in 1 - to 2 -week-old DKO mice of both sexes. A progressive loss of cells containing these proteins occurred as animals aged, however (Figure 4). Eight-week-old hyperglycemic male mice exhibited a marked reduction in insulin-positive cells: in some cases, only a few scattered cells could be found. The number of glucagon-producing cells was normal or near normal for a longer period of time, although older, sick mice exhibited few glucagon-producing cells, which seemed to be sufficient to maintain normal glucagon levels in the serum of hyperglycemic DKO animals. Finally, the overall content of $\alpha$-amylase was significantly reduced in adult DKO animals, consistent with the lower enzymatic activity found in the serum of these animals.

We also investigated whether the allocation of specific cell types during development was deregulated in DKO mice by analyzing the expression of transcription factors responsible for pancreas development and differentiation. The expression of Pdx-1, Pax-6, and Isl-1 was similar at postnatal day 7 in DKO and WT mice (Figure 5), arguing that pancreatic development and cell specification were unaffected.

Increased proliferation and apoptosis in DKO pancreatic cells. We examined cellular proliferation and apoptosis in the pancreas of DKO mice and littermate controls. BrdU was injected into male and female DKO and WT animals, and S-phase cells were detected with an $\mathrm{Ab}$ to BrdU. Pancreatic cells divide actively during the first week of postnatal age, but proliferation decreases by the second week, concomitant with maturation of the pancreas (24). Consistent with this, a small proportion of $\mathrm{BrdU}^{+}$cells were present in the pancreas of 2-week-old WT mice (Figure 6; Table 1). Surprisingly, when we examined 2-week-old or 4-week-old E2F1/ E2F2 double-deficient mice, we found that a significant fraction of the cells were still replicating DNA, and many of the $\mathrm{BrdU}^{+}$ cells were those with aberrantly large nuclei (Figure 6; Table 1). BrdU-positive cells were found both in the exocrine as well as in the endocrine compartment. In absolute terms, the vast majority of the cells undergoing DNA replication were exocrine cells $(96 \% \pm 9.3 \%)$, although a fraction of $\mathrm{BrdU}^{+}$cells was also found within the islets. In relative terms, $1-3 \%$ of the exocrine cells and $0.5-1 \%$ of the endocrine cells were $\mathrm{BrdU}^{+}$. To examine apoptosis, the TUNEL assay was performed. The proportion of apoptotic nuclei detected by TUNEL was also clearly increased in 2- and 4week-old DKO mice (Figure 6; Table 1). These results suggest that loss of E2F1 and E2F2 results in unscheduled DNA replication and induction of apoptosis.

We next examined the individual contribution of E2F1 and E2F2 to the deregulated cell cycle found in pancreata of 2- and 4-weekold DKO mice. The number of cells undergoing DNA synthesis was significantly higher in $E 2 \mathrm{~F}^{-/-}$mice than in age-matched WT mice (Table 1). DNA synthesis was similar to that of WT mice in male $E 2 F 1^{-/-}$pancreata, however, and slightly higher in female $E 2 \mathrm{~F}^{-/-}$pancreata (Table 1). Contrary to what was observed in DKO mice, the large, aberrant nuclei present in E2F1-deficient mice did not incorporate BrdU. Increased numbers of apoptotic cells were observed in $\mathrm{E}_{2} \mathrm{~F}^{-/-}$pancreata but not in $\mathrm{E} 2 \mathrm{~F} 2^{-/-}$pancreata (Table $1)$. These results argue that the severe pancreatic phenotype exhibited by DKO mice is not simply a dosage effect resulting from the addition of two similar, albeit milder, phenotypes present in individual $E 2 \mathrm{F1}^{-{ }^{-}}$and $E 2 \mathrm{F2}^{-{ }^{-}}$mice and suggest that each $E 2 F$ gene plays a specific role in pancreatic homeostasis. Rather, it appears that loss of E2F1 results in large aberrant nuclei and increased apoptosis, while loss of E2F2 results in increased cell-cycle entry. The combination of these effects in DKO mice in some way results in postnatal involution of the pancreas.

Differential gene expression in DKO pancreatic cells. To gain insight into E2F1/E2F2-regulated gene expression in the pancreas we made use of high-density Affymetrix GeneChip oligonucleotide microarrays and analyzed the expression profile of approximately 12,000 murine gene sequences and expressed sequence tags (ESTs) in pancreatic cells isolated from 2-week-old DKO mice compared with age-matched WT mice. This time point was chosen because at this age the DKO pancreata appeared histologically normal. We identified approximately 300 genes and ESTs whose expression was altered at least twofold in DKO cells relative to WT cells. Of these, 172 corresponded to annotated genes, 155 of which were upregulated and 17 downregulated (see supplemental material available at http://www.jci.org/cgi/content/ full/113/10/1398/DC1.

Remarkably, a significant number of genes previously identified as components of the $\mathrm{E} 2 \mathrm{~F} / \mathrm{Rb}$ regulatory pathway were found to be overexpressed more than twofold in the pancreata of 2-week-old DKO mice. These genes could be clustered into functional groups, previously classified by Ren and collaborators (7), related to cell cycle regulation, DNA replication, DNA repair, mitotic checkpoint, and chromosome transactions (cyclin D2, mcm's cdc-6-related protein, PCNA, MAD1, septin) $(5,7)$. Expression of the proapoptotic gene Bax was also increased in DKO samples. Other genes with increased expression in the DKO pancreas include those encoding cytoskeletal and ECM proteins, inflammatory proteins, and enzymes involved in fatty-acid metabolism, suggesting an aberrant metabolic state of these cells. Markers of ductal cells (keratin-19, carbonic anbydrase) and adipocytes (adipsin, Acrp30) were also overexpressed in DKO pancreata.

By contrast, the number of genes whose expression was reduced at least 50\% upon E2F1 and E2F2 inactivation was low. Of those, genes encoding proteins involved in exocrine and endocrine function constituted $50 \%$ of all downregulated sequences (trypsin, $\alpha$-amylase, Reg's, IGF-1). The expression of insulin mRNA was also 
downregulated in 2-week-old DKO animals (35\% reduction relative to WT mice), but not that of glucagon or somatostatin (data not shown). Taken together, these results suggest that the combined E2F1/E2F2 activity may be involved in negatively regulating proliferation and in promoting and/or maintaining the terminal differentiation of pancreatic cells.

\section{Discussion}

Our studies reveal that E2F1 and E2F2 play a critical role in the maintenance of pancreatic homeostasis. E2F1/E2F2 compoundmutant mice have excessive polyuria and hyperglycemia. The marked decrease in blood insulin levels and in the proportion of insulin-expressing pancreatic cells indicates that diabetes results from a deficit in insulin production. The lack of pancreatic inflammatory infiltrates in hyperglycemic animals (Figure $2, \mathrm{E}-\mathrm{G}$ ) and the inability of the immunosuppressant sodium fusidate to lower the glycemia of hyperglycemic DKO mice at a dose known to reduce murine inflammatory diabetes (25) argue against an autoimmune nature of the diabetes (data not shown). The presence of steatorrhea, the decrease in serum amylase activity, as well as the histological findings, indicate a concomitant exocrine insufficiency. Interestingly, the diabetic phenotype is more severe in male mice than in female mice lacking E2F1 and E2F2, similar to what has been reported in other animal models such as Cdk4-deficient mice (26) or Akt2-deficient mice (27); it suggests that the control of glucose metabolism in these mice is finely tuned by gender-specific factors.

Recent studies suggest that E2F may directly regulate developmental genes through a mechanism that is independent of the cell cycle (3). A Xenopus E2F gene has been identified that is most closely related to E2F3, which has been found to control anteriorposterior axis formation in Xenopus (28). Thus, we postulated that the defects in E2F1/E2F2 compound-mutant mice could result from aberrant pancreas development and differentiation. Several genes encoding transcription factors that exert fundamental roles in pancreas development and differentiation have been identified, including PDX1, Mist1, Pax6, Isl1, and $p 48(29,30)$. The roughly normal histology of the DKO pancreas at birth, and the lack of alterations in the expression of these genes in the pancreatic lineages, however, suggest that cell-type specification is not affected and that these transcription factors are not primarily involved in the pancreatic defects of adult DKO mice.

Dissecting the mechanisms underlying the disruption of pancreatic homeostasis in E2F1/E2F2 double-mutant mice has proven complex, since $E 2 F 1$ and $E 2 F 2$ genes are involved in the regulation of multiple processes. First, young DKO mice display a marked increase in DNA synthesis rate in the pancreas. This is accompanied by an increase in apoptosis, eventually leading to pancreatic atrophy. Finally, acinar cells show marked hypertrophy and dysplasia, and islets essentially disappear. Although it is unclear how the increased DNA replication is related to the increased apoptosis in the pancreas of DKO mice, it has been reported that deregulation of DNA replication can result in chromosome instability and induction of cell death in other systems $(31,32)$, and recent work by DeGregori and colleagues suggests that the increased apoptosis observed in hematopoietic progenitors upon concomitant inactivation of E2F1 and E2F2 is a consequence of inappropriate $\mathrm{S}$-phase progression and of blocked B cell maturation (33). In the pancreas, E2F1 and E2F2 could have a role in preventing the cells from entering the $\mathrm{S}$ phase during the maturation of the organ. Loss of E2F1 and E2F2 would result in unscheduled G1/S progression, which could, in turn, activate an S-phase checkpoint and induce apoptosis. The analysis of $E 2 \mathrm{~F}^{-/-}$and $E 2 \mathrm{~F}^{-/-}$single-mutant mice suggests that each E2F member contributes in a nonredundant fashion to normal pancreatic function: E2F1 may help maintain normal nuclear structure, as suggested by Yamasaki and collaborators (12), whereas E2F2 may negatively regulate pancreatic cellular proliferation, as occurs in $\mathrm{T}$ lymphocytes (15). The combination of two altered phenotypes and the loss of compensation in double homozygote mice may account for an increased apoptotic rate and a severe involution of this tissue.

Remarkably, many cell cycle-related genes, some of them known E2F targets, appear to be negatively regulated by E2F1/E2F2 in pancreatic tissue, implying that their increased expression due to loss of E2F1/E2F2 activity could lead to the unchecked proliferation and apoptosis operating in DKO pancreata. Many of the genes identified as downregulated by the E2F1/2 proteins include those encoding DNA replication activities (PCNA; $\mathrm{mcm}$ $3,5,7 ; c d c-6)$, and G1-regulated genes (cyclin G1, cyclin D2), suggesting that E2F1/2 play crucial roles in the negative regulation of $\mathrm{G} 1 / \mathrm{S}$ progression in the pancreas. This role is important because this tissue is essentially quiescent in the adult, and there is extensive evidence from the analysis of pancreatic cancer that the p53 and $\mathrm{Rb}$ pathways are essential for growth control $(34,35)$. Overproduction of proteins encoded by genes involved in origin firing and in $\mathrm{G} 1 / \mathrm{S}$ progression, such as those found in the microarray analysis of E2F1/E2F2 DKO pancreata, may force the cells to enter $S$ phase inappropriately and activate an S-phase checkpoint. Ectopic expression of a deregulated form of Swi-4, a G1-specific transcriptional activator, results in precocious S-phase entry and activation of a Rad53-dependent checkpoint (31). Increased expression of the p53 target gene Bax in DKO pancreata is consistent with the activation of a checkpoint. Finally, consistent with previous work, a number of $\mathrm{E} 2 \mathrm{~F} 1 / 2$ responsive genes in the pancreas, including septin 2 and septin 7 , are typically regulated at $\mathrm{G} 2 / \mathrm{M}$ in the cell cycle and encode proteins that function in mitosis $(5,7)$. Although upregulation of these genes could result from perturbations in $\mathrm{G} 1 / \mathrm{S}$ progression, it is also possible that E2F1 and E2F2 control the expression of mitotic genes directly, because direct binding of $\mathrm{E} 2 \mathrm{~F}$ proteins to the promoter sites of some of these genes has been reported (7). It remains to be determined which of these regulatory effects are direct.

Intriguingly, E2F1 and E2F2 have been traditionally included in a subclass of E2Fs considered to be transcriptional activators, unlike the repressors E2F4/5 (36-39). In promoter occupancy assays, E2F1 and E2F3 were enriched at promoters activated during the G1-to-S-phase transition, whereas E2F4 was recruited to promoters repressed in quiescent cells $(9,10)$. Furthermore, analyses of mice deficient in E2F4 or E2F5 are consistent with a role for these two E2F family members in regulating cell commitment in several cell types $(18,19)$. Our results on T lymphocyte hyperproliferation challenged the notion that E2F2 is a transcriptional activator by showing that E2F2 can repress the transcription of promoters containing E2F sites (15). The findings reported here further emphasize that E2F1/2 may function as negative regulators of gene expression, much the same way as the group including E2F4/5, implying that these two groups of E2F proteins may play similar roles in mediating cell cycle arrest and allowing cell differentiation in a tissue-specific fashion, either directly or indirectly. 
Although the pathological features exhibited by the E2F1/E2F2 DKO pancreas are unlike those reported in other genetically modified mice or in humans, DKO mice display several features of chronic pancreatitis. In this disease, there is also loss of acinar cells, accompanied by extensive tissue fibrosis, inflammation, fat replacement, and ductal metaplasia, leading to exocrine insufficiency. Abnormal activation and secretion of proteolytic enzymes, mainly trypsin, may play a pivotal role in this process (40). Both in mice and humans, chronic pancreatic insufficiency is associated with reduced acinar enzyme serum levels. Despite these similarities, two histological features of the pancreas of E2F1/E2F2 double homozygotes occur rarely in chronic pancreatitis: the severe acinar cell hypertrophy and dysplasia and the almost complete disappearance of islets. Furthermore, Reg genes, which are typically overexpressed in pancreatitis $(41,42)$, were downregulated in pancreata of DKO mice. Likewise, inflammatory infiltrates were not seen in the involuting DKO pancreata, and treatment with immunosuppressants failed to rescue the pancreata in these mice (data not shown).

Presently, it is unclear whether the exocrine and the endocrine involution of the DKO pancreas represents a single process or two separate processes. Retroviral vector lineage tracing has demonstrated that exocrine and endocrine cells share a common ductal progenitor during development (43). Furthermore, studies on cell renewal and differentiation in the adult pancreas have shown that pancreatic ductal cells have the capacity to proliferate and differentiate terminally into both endocrine and exocrine lineages, recapitulating embryonic development (44). In addition, acinar-ductal transdifferentiation occurs frequently in chronic pancreatitis and occurs in vitro upon culture of normal exocrine pancreatic cells (45). In E2F1/E2F2 DKO mice, loss of the exocrine and endocrine components is associated postnatally with an increase in cells with a ductal-like morphology containing zymogen - and sometimes endocrine - granules, an increase in ductal structures in both compartments, and a marked downregulation of pancreas-specific genes together with an upregulation of ductal-specific genes, suggesting that in DKO mice there is a defect in the maintenance of the differentiated pancreatic phenotype. Thus, we would like to propose that E2F1 and E2F2 are required postnatally for the maintenance of the endocrine and exocrine compartments. Further analysis on the temporal and cell type-specific expression patterns of E2F1 and E2F2 in the pancreas and the selective inactivation of these genes in the exocrine and endocrine lineages will help establish their precise role in pancreatic cell homeostasis.

Note added in proof. While this work was under review, a paper entitled "The development of diabetes in E2f1/E2f2 mutant mice reveals important roles for bone marrow-derived cells in preventing islet cell loss" was published by DeGregori and colleagues (46). Their results are complementary and in agreement with those presented in this manuscript, thus supporting the idea that E2F1 and E2F2 transcription factors are essential for pancreatic homeostasis.

\section{Acknowledgments}

This work was supported by the following grants: BMC2000-1131 from the Spanish Ministry of Science and Technology, 9/UPV 00154.310-13554/2001 from the University of the Basque Country, IE01UN12 from the Basque Government (to A.M. Zubiaga), 99-3010 from Marató TV-3, and SAF2001-0420 and GEN20014748-C05-01 from the Spanish Ministry of Science and Technology (to F.X. Real). A. Iglesias is a recipient of a Spanish Ministry of Education fellowship for graduate studies. M. Murga and U. Laresgoiti are recipients of Basque Government fellowships for graduate studies. S.J. Field is a Howard Hughes Medical Institute postdoctoral fellow. We are grateful to Carmen Mar and Begoña Ochoa for determination of plasma insulin, glucagon, ion, and metabolite levels. We would like to thank Marcos Malumbres for experimental suggestions and interpretations, Oskar FernándezCapetillo for his help on the initial experimental setup, Xavier Mayol for critical reading of this manuscript, and members of the lab for technical assistance and helpful discussions.

Received for publication May 8, 2003, and accepted in revised form March 16, 2004.

Address correspondence to: A.M. Zubiaga, Department of Genetics, Physical Anthropology and Animal Physiology, Faculty of Sciences, University of the Basque Country, Apartado 644, Bilbao E-48080, Spain. Phone: 34-94-601-2603; Fax: 34-94-464-8500; E-mail: ggpzuela@lg.ehu.es.

Matilde Murga's present address is: Experimental Transplantation and Immunology Branch, Center for Cancer Research, National Cancer Institute, National Institutes of Health, Bethesda, Maryland, USA.

\footnotetext{
1. Boyd, K.E., and Farnham, P.J. 1999. Identification of target genes of oncogenic transcription factors. Proc. Soc. Exp. Biol. Med. 222:9-28.

2. Dyson, N. 1998. The regulation of E2F by pRbfamily proteins. Genes Dev. 12:2245-2262.

3. Helin, K. 1998. Regulation of cell proliferation by the E2F transcription factors. Curr. Opin. Genet. Dev. 8:28-35.

4. DeGregori, J. 2002. The genetics of the E2F family of transcription factors: shared functions and unique roles. Biochim. Biophys. Acta. 1602:131-150.

5. Ishida, S., et al. 2001. Role for E2F in control of both DNA replication and mitotic functions as revealed from DNA microarray analysis. Mol. Cell. Biol. 21:4684-4699.

6. Müller, H., et al. 2001. E2Fs regulate the expression of genes involved in differentiation, development, proliferation, and apoptosis. Genes Dev. 15:267-285.

7. Ren, B., et al. 2002. E2F integrates cell cycle progression with DNA repair, replication, and G2/M checkpoints. Genes Dev. 16:245-256.
}

8. Weinmann, A.S., Yan, P.S., Oberley, M.J., Huang, T.H., and Farnham, P.J. 2002. Isolating human transcription factor targets by coupling chromatin immunoprecipitation and $\mathrm{CPG}$ island microarray analysis. Genes Dev. 16:235-244.

9. Takahashi, Y., Rayman, J.B., and Dynlacht, B.D. 2000. Analysis of promoter binding by the E2F and pRB families in vivo: distinct E2F proteins mediate activation and repression. Genes Dev. 14:804-816.

10. Wells, J., Boyd, K.E., Fry, C.J., Bartley, S.M., and Farnham, P.J. 2000. Target gene specificity of E2F and pocket protein family members in living cells. Mol. Cell. Biol. 20:5797-5807.

11. Field, S.J., et al. 1996. E2F1 functions in mice to promote apoptosis and suppress proliferation. Cell. 85:549-561.

12. Yamasaki, L., et al. 1996. Tumor induction and tissue atrophy in mice lacking E2F-1. Cell. 85:537-548.

13. Zhu, J.W., DeRyckere, D., Li, F.X., Wan, Y.Y., and DeGregori, J. 1999. A role for E2F1 in the induction of ARF, p53, and apoptosis during thymic negative selection. Cell Growth Differ. 10:829-838.

14. García, I., Murga, M., Vicario, A., Field, S.J., and Zubiaga, A.M. 2000. A role for E2F1 in the induction of apoptosis during thymic negative selection. Cell Growth Differ. 11:91-98.

15. Murga, M., et al. 2001. Mutation of E2F2 in mice causes enhanced $\mathrm{T}$ lymphocyte proliferation, leading to the development of autoimmunity. Immunity. 15:959-970.

16. Zhu, J.W., et al. 2001. E2F1 and E2F2 determine thresholds for antigen-induced T-cell proliferation and suppress tumorigenesis. Mol. Cell. Biol. 21:8547-8564.

17. Humbert, P.O., et al. 2000. E2F3 is critical for normal cellular proliferation. Genes Dev. 14:690-703.

18. Humbert, P.O., et al. 2000. E2F4 is essential for normal erythrocyte maturation and neonatal viability. Mol. Cell. 6:281-291.

19. Rempel, R.E., et al. 2000. Loss of E2F4 activity leads to abnormal development of multiple cellular lineages. Mol. Cell. 6:293-306. 
20. Lindeman, G.J., et al. 1998. A specific, nonproliferative role for E2F-5 in choroids plexus function revealed by gene targeting. Genes Dev. 12:1092-1098.

21. Wells, J., Graveel, C.R., Bartley, S.M., Madore, S.J., and Farnham, P.J. 2002. The identification of E2F1specific target genes. Proc. Natl. Acad. Sci. U. S. A. 99:3890-3895

22. Cloud, J.E., et al. 2002. Mutant mouse models reveal the relative roles of E2F1 and E2F3 in vivo. Mol. Cell Biol. 22:2663-2672.

23. Wu, L., et al. 2001. The E2F1-3 transcription factors are essential for cellular proliferation. Nature. 414:457-462.

24. Heller, R.S., et al. 2001. Improved glucose tolerance and acinar dysmorphogenesis by targeted expression of transcription factor PDX-1 to the exocrine pancreas. Diabetes. 50:1553-1561.

25. Nicoletti, F., et al. 2000. Sodium fusidate ameliorates the course of diabetes induced in mice by multiple low doses of streptozotocin. J. Autoimmun. 15:395-405.

26. Rane, S.G., et al. 1999. Loss of Cdk4 expression causes insulin-deficient diabetes and Cdk 4 activation results in $\beta$-islet cell hyperplasia. Nat. Genet. 22:44-52.

27. Garofalo, R.S., et al. 2003. Severe diabetes, agedependent loss of adipose tissue, and mild growth deficiency in mice lacking Akt2/PKB $\beta$. J. Clin. Invest. 112:197-208. doi:10.1172/JCI200316885.

28. Suzuki, A., and Hemmati-Brivanlou, A. 2000. Xenopus embryonic E2F is required for the formation of ventral and posterior cell fates during early embryogenesis. Mol. Cell. 5:217-229.
29. Edlund, H. 1998. Transcribing pancreas. Diabetes. 47:1817-1823.

30. Kawaguchi, Y., et al. 2002. The role of the transcriptional regulator Ptf1a in converting intestinal to pancreatic progenitors. Nat. Genet. 32:128-134.

31. Sidorova, J.M., and Breeden, L.L. Precocious S-phase entry in budding yeast prolongs replicative state and increases dependence upon Rad53 for viability. Genetics. 160:123-136.

32. Watanabe, K., Morishita, J., Umezu, K., Shirahige, K., and Maki, H. 2002. Involvement of Rad9-dependent damage checkpoint control in arrest of cell cycle, induction of cell death, and chromosome instability caused by defects in origin recognition complex in Saccharomyces cerevisiae. Eukaryot. Cell. 1:200-212.

33. Li, F.X., Zhu, J.W., Hogan, C.J., and DeGregori, J. Defective gene expression, $\mathrm{S}$ phase progression, and maturation during hematopoiesis in E2F1/ E2F2 mutant mice. Mol. Cell. Biol. 23:3607-3622.

34. Rozenblum, E., et al. 1997. Tumor-suppressive pathways in pancreatic carcinoma. Cancer Res. 57:1731-1734.

35. Bardeesy, N., and DePinho, R.A. 2002. Pancreatic cancer biology and genetics. Nat. Rev. Cancer. 2:897-909.

36. Johnson, D.G., Schwarz, J.K., Cress, W.D., and Nevins, J.R. 1993. Expression of transcription factor E2F1 induces quiescent cells to enter S phase. Nature. 365:349-352.

37. Lees, J.A., et al. 1993. The retinoblastoma protein binds to a family of E2F transcription factors. $\mathrm{Mol}$. Cell. Biol. 13:7813-7825.

38. Lukas, J., Petersen, B.O., Holm, K., Bartek, J., and
Helin, K. 1996. Deregulated expression of E2F family members induces S-phase entry and overcomes p16INK4A-mediated growth suppression. Mol. Cell. Biol. 16:1047-1057.

39. Harbour, J.W., and Dean, D.C. 2000. The Rb/E2F pathway: expanding roles and emerging paradigms. Genes Dev. 14:2393-2409.

40. Whitcomb, D.C. 2001. Hereditary pancreatitis: a model for understanding the genetic basis of acute and chronic pancreatitis. Pancreatology. 1:565-570.

41. Zenilman, M.E., Tuchman, D., Zheng, Q., Levine, J., and Delany, H. 2000. Comparison of reg I and reg III levels during acute pancreatitis in the rat. Ann. Surg. 232:646-652.

42. Graf, R., Schiesser, M., Lussi, A., Went, P., Scheele, G.A., and Bimmler, D. 2002. Coordinate regulation of secretory stress proteins (PSP/reg, PAP I, PAP II, and PAP III) in the rat exocrine pancreas during experimental acute pancreatitis. J. Surg. Res. 105:136-144.

43. Fishman, M.P., and Melton, D.A. 2002. Pancreatic lineage analysis using a retroviral vector in embryonic mice demonstrates a common progenitor for endocrine and exocrine cells. Int. J. Dev. Biol. 46:201-207.

44. Bonner-Weir, S., and Shama, A. 2002. Pancreatic stem cells. J. Pathol. 197:519-526.

45. Vilá, M.R., Lloreta, J., and Real, F.X. 1994. Normal human pancreas cultures display functional ductal characteristics. Lab. Invest. 71:423-431.

46. Li, F.X., et al. 2003. The development of diabetes in E2f1/E2f2 mutant mice reveals important roles for bone marrow-derived cells in preventing islet cell loss. Proc. Natl. Acad. Sci. U.S.A. 100:12935-12940. 